Yonathan Freund Camille Chenevier-Gobeaux Yann-Erick Claessens François Leumani Benoit Doumenc Pascale Bonnet Jean-Christophe Allo Claudine Cosson Bruno Riou Patrick Ray

\section{RETRACTED ARTICLE: Concomitant measurement of copeptin and high-sensitivity troponin for fast and reliable rule out of acute myocardial infarction}

Received: 26 April 2011

Accepted: 19 December 2011

Published online: 1 February 2012

(C) Copyright jointly held by Springer and ESICM 2012
Y. Freund $(\bowtie) \cdot$ B. Riou

Service d'Accueil des Urgences,

Hôpital Pitié-Salpétrière,

Assistance Publique-Hôpitaux de Paris

(APHP), Université Pierre et Marie

Curie-Paris 6 (UPMC),

47-83 Boulevard de l'Hôpital,

75651 Paris cedex 13, France

e-mail: yonathanfreund@gmail.com

Tel.: +33-1-42177912

Fax: +33-1-42177412
C. Chenevier-Gobeaux Departement de Biochimie, Hôpital Cochin-Hôtel Dieu, APHP, 27 rue du Faubourg Saint-Jacques, 75679 Paris cedex 14, France

\section{Y.-E. Claessens · J.-C. Allo}

Service d'Accueil des Urgences, Hôpital Cochin-Hôtel Dieu, APHP, Université Paris Descartes-Paris 5, 27 rue du Faubourg Saint-Jacques, 75679 Paris cedex 14, France

\section{B. Doumenc}

Service d'Accueil des Urgences, Hôpital Bichat, APHP, 46 Rue Henri Huchard, 75018 Paris, France

C. Cosson

Departement de Biochimie, Hôpital Bicêtre, APHP, 78 rue du Général Leclerc, 94270 Le Kremlin-Bicêtre, France
B. Riou · P. Ray

INSERM UMRS 956, UPMC,

91 Boulevard de l'Hôpital,

75013 Paris, France

F. Leumani $\cdot$ P. Bonnet

Service d'Accueil des Urgences,

Hôpital Pitié-Salpétrière, APHP,

47-83 Boulevard de l'Hôpital,

75651 Paris cedex 13, France

P. Ray

Service d'Accueil des Urgences, Hôpital Tenon, APHP, UPMC, 4 rue de la Chine,

75970 Paris cedex 20, France

This article has been retracted due to copyright violation. 